\title{
Mechanisms Contributing to Malignant Dysrhythmias Induced by Ischemia in the Cat
}

\author{
Peter B. Corr, Francis X. Witkowski, and Burton E. Sobel, The Cardiovascular \\ Division, Washington University School of Medicine, St. Louis, Missouri 63110
}

\begin{abstract}
A B S T RACT Continuously recorded bipolar electrograms were obtained simultaneously from epi-, endo-, and mid-myocardial regions of the ischemic and normal zones of cat left ventricle in vivo after coronary occlusion, analyzed by computer, and compared to regional cyclic AMP levels. Regional cyclic AMP content was used as an index of the combined local effects of: $(a)$ efferent sympathetic nerve discharge; $(b)$ release of myocardial catecholamines due to ischemia; and $(c)$ circulating catecholamines. Ischemia resulted in a progressive increase in pulse width and rise time and a decrease in rate of rise of voltage $(\mathrm{d} V / \mathrm{d} t)$ of the local electrograms from ischemic zones reaching a maximum within $2.4 \pm 0.3 \mathrm{~min}($ mean $\pm \mathrm{SE})$ at the time of onset of severe ventricular dysrhythmias, all of which returned toward control before the cessation of the dysrhythmia $(33.5 \pm 1.5 \mathrm{~min}$ after coronary occlusion). Increases in cyclic AMP in ischemic zones preceded corresponding increases in the frequency of premature ventricular complexes (PVCs). Propranolol inhibited the increases in cyclic AMP and reduced the frequency of PVCs in animals without ventricular fibrillation. In animals with ventricular fibrillation, cyclic AMP was significantly elevated in normal and ischemic zones compared to animals with PVCs only. Electrical induction of PVCs or ventricular fibrillation in ischemic and nonischemic hearts failed to increase cyclic AMP. The results suggest that the changes in regional adrenergic stimulation of the heart may contribute to perpetuation of ventricular dysrhythmia and the genesis of ventricular fibrillation early after the onset of myocardial ischemia.
\end{abstract}

\section{INTRODUCTION}

In experimental animals, dysrhythmias occurring soon after the onset of myocardial ischemia differ markedly

A preliminary report was presented at the 1977 Annual Meeting of the American Society for Clinical Investigation, Washington, D. C.

Received for publication 11 May 1977 and in revised form 6 September 1977. from the relatively benign dysrhythmias occurring later (1-5). Thus, elucidation of factors contributing to dysrhythmias associated with sudden death in man may require analysis of processes occurring during the interval immediately after the onset of myocardial ischemia.

Several observations implicate deleterious sympathetic nervous system influences on these early dysrhythmias. Among patients seen within $30 \mathrm{~min}$ after the onset of acute myocardial ischemia (6), prominent alterations in the activity of the autonomic nervous system occur in $92 \%$. Complete myocardial catecholamine depletion (i.e., after mediastinal neural ablation) confers striking protection against ventricular fibrillation in animals (7). However, prior treatment with propranolol in doses sufficient to attenuate the response to maximal stellate ganglion stimulation does not confer protection against ventricular fibrillation (8, 9 ). This may be due to incomplete obliteration of neural as opposed to humoral sympathetic input to the heart by pharmacological blockade (10) or $\alpha$-receptor contributions to the malignant dysrhythmias induced by ischemia (11).

Sympathetic nerve recordings during myocardial ischemia may not reflect alterations in sympathetic input to selected regions of myocardium because of extensive arborization of the nerves distal to the recording site as well as additional influences of circulating catecholamines. Measurement of local myocardial catecholamines may not reflect regional efferent sympathetic activity because of the combined loci of norepinephrine in peripheral nerve terminals and cardiac effector cells. Incompleteness of pharmacological blockade or intramyocardial release of norepinephrine may cloud the interpretation of results based solely on changed global function after $\beta$-receptor blockade.

For these reasons, in the present study we assayed regional myocardial cyclic adenosine $3^{\prime}, 5^{\prime}$-monophosphate (cyclic AMP) content as one index of regional adrenergic activity, based on the concept that transient changes in myocardial cyclic AMP content may reflect 
regional postsynaptic influences of catecholamines (12-14). Accordingly, measurement of regional cyclic AMP content may permit assessment of the combined neural, humoral, and intramyocardial effects of catecholamines in selected regions of the heart. Although factors other than catecholamines may contribute to changes in cyclic AMP, including alterations in phosphodiesterase activity (15), effects of anoxia per se (16-18), or decreased utilization and (or) washout of cyclic AMP, most of the increase in cyclic AMP in anoxic or ischemic hearts has been shown to reflect adrenergic stimulation rather than direct effects of anoxia $(17,18)$.

In the present study, we utilized an experimental animal preparation exhibiting consistent ventricular dysrhythmia early after coronary occlusion to determine: (a) whether specific changes in local electrograms in ischemic tissue correlate with the onset or spontaneous termination of ventricular dysrhythmia; (b) whether characteristic changes occurred in regional adrenergic activity, reflected by increased cyclic AMP content in ischemic and nonischemic zones before, during, and after termination of ventricular dysrhythmia; and $(c)$ whether alterations in cyclic AMP content after ischemia depend on regional adrenergic influences based on results after $\beta$-adrenergic blockade.

\section{METHODS}

Animal preparation. Male and female adult cats ( $n$ $=157)(2.1-3.9 \mathrm{~kg})$ were anesthetized with $\alpha$-chloralose $(75 \mathrm{mg} / \mathrm{kg}$ ) to minimize depression of cardiac reflexes by anesthesia. After endotracheal intubation, each animal was mechanically ventilated with a mixture of oxygen and room air to maintain normal arterial $\mathrm{pH}, \mathrm{PCO}_{2}$ and $\mathrm{PO}_{2}$ monitored every $5 \mathrm{~min}$ in several animals. Adjustment of tidal volumes $\left(\cong 20 \mathrm{~cm}^{3} / \mathrm{kg}\right.$ ) resulted in maintenance of normal $\mathrm{pH}$ and blood gas values. Left thoracotomy was performed by excision of ribs two through four, and body temperature was maintained at $37.5^{\circ} \mathrm{C}$ with the use of a thermostatic esophageal probe controlling an infrared lamp. The pericardium was open and sutured to the chest wall to form a cradle, and the left anterior descending coronary artery was isolated at its bifurcation from the main left trunk, proximal to all branch points under microscope visualization. A Gould-Brush model 260 recorder (Gould, Inc., Instrument Systems Div., Cleveland, Ohio) was used to monitor multiple lead surface electrocardiograms and systemic arterial pressure via a femoral artery catheter.

Bipolar electrogram recordings and automated analysis. Bipolar electrograms from epi-, myo-, and subendocardial areas of ischemic and normal zones of the left ventricle were obtained as follows: The epicardial electrodes consisted of two gold disks ( $1.5 \mathrm{~mm}$ diameter and 0.004 inch thickness) soldered to fine stainless steel wires coated with polyurethane, imbedded in a pliable silicone mat and sutured to the epicardial surface. The myocardial and subendocardial electrodes were made from four polyurethane-coated stainless steel wires which were bonded together with an additional coat of polyurethane. The two subendocardial leads were turned at right angles to form a hook and the tips were cut to provide electrical contact. The two myocardial leads were cut $3.5 \mathrm{~mm}$ proximal to the beveled end of the subendocardial electrode to provide a mid-myocardial recording. The entire electrode bundle was checked for continuity and lack of crossover between leads with an ohmmeter and subsequently placed in a 25-gauge needle and inserted through the left ventricular wall immediately adjacent to the epicardial electrode. The needle was withdrawn so that the subendocardial leads engaged the inner wall and the myocardial electrode engaged the myocardium at the mid wall level. Each of the six bipolar electrode contacts (in both ischemic and normal zones) were placed in multiple silver-contact electrode holders, amplified by a constant gain AC amplifier and variable gain DC amplifier-universal coupler (Gould Inc.) with low frequency cutoff $(3 \mathrm{~dB})$ at $1.0 \mathrm{~Hz}$ and high frequency cutoff $(3 \mathrm{~dB})$ at $1.0 \mathrm{kHz}$ before storage on FMmagnetic tape and display on an eight-channel monitor oscilloscope. Zero level (short-circuit of the amplifier input lines) and calibration level (constant $1 \mathrm{mV}$ ) were also recorded on FM-magnetic tape for subsequent calibration of the waveforms. The location of each electrode was verified at the termination of each experiment. Variance in electrode placement from experiment to experiment was minimized by placing the ischemic zone electrodes $3.0 \mathrm{~mm}$ lateral to the left anterior descending coronary artery just distal to the first medial branch (mid-ischemic zone) and placing the normal zone electrode on the lateral left ventricular wall adjacent to the first major branch of the left circumflex coronary artery.

Automated analysis of the bipolar electrograms was performed in real time with a PDP-12 computer system and interactive controlling software written in LINC/PDP-8 Assembly language. The system digitizes the analog signal at a $10-\mathrm{kHz}$ sampling rate. When a signal equal to or in excess of threshold is identified, the program retains the 200 samples before the time when the threshold requirement is fulfilled along with the 300 subsequent samples providing a total of 500 data points $(50 \mathrm{~ms})$ per pulse. The following parameters are then obtained from each captured bipolar electrogram: (a) maximum (MAX $=100 \%$ ) and minimum (MIN $=0 \%$ - the highest absolute voltage detected after threshold and the lowest detected within the 20 -ms preceding threshold; (b) the difference (millivolt) between MAX and MIN; $(c)$ the rise time (the interval (millisecond) during which voltage increases from 25 to $75 \%$ of MAX-MIN); (d) the pulse width at $50 \%$ amplitude (millisecond); and (e) the rate of rise of voltage (millivolt/millisecond) from 25 to $75 \%$ of $\mathrm{MAX}-\mathrm{MIN} /(\mathrm{dV} / \mathrm{d} t)$.

Acquisition of data occurs during each systole and analysis during diastole. Thus, as soon as each electrogram has been analyzed, acquisition is reinstituted automatically. The system calculates the mean and SD for each derived parameter and displays all parameters graphically as a function of time utilizing a Houston Instruments (Div. Bausch \& Lomb, Inc., Austin, Tex.) DP1-5H incremental plotter.

Biochemical procedures. Cyclic AMP content in normal and central ischemic zones of the left ventricle was assessed at selected intervals after coronary occlusion in fast-frozen biopsies $(\cong 200 \mathrm{mg}$ ) obtained with a suction-drill through the left ventricular wall from normal and ischemic zones. One pair of samples was obtained from each cat after which the experiment was terminated. Biopsy samples, stored at $-80^{\circ} \mathrm{C}$ (REVCO, Inc., West Columbia, S.C.) were pulverized in a steel mortar frozen in liquid nitrogen, quickly transferred to a cold homogenizer cup containing $0.75 \mathrm{ml}$ of $6 \%$ trichloroacetic acid, thoroughly homogenized (Omni Mini- 


\section{SURFACE ECG LEAD II}

CONTROL Before Occlusion
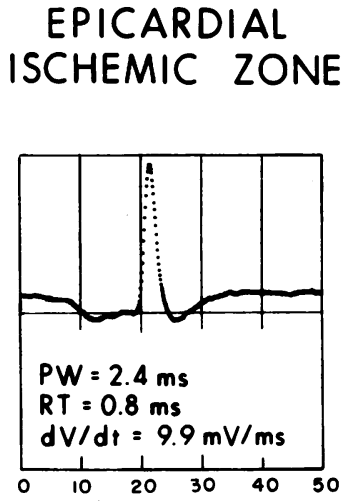

EPICARDIAL NORMAL ZONE

\section{time in $\mathrm{ms}$}

\subsection{Min after Occlusion at Onset of Dysrhythmia}
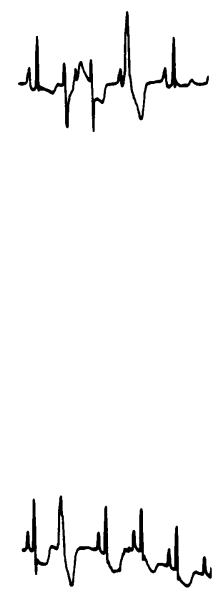
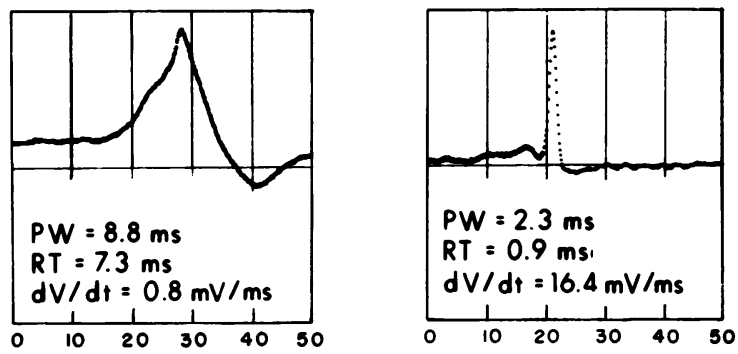

time in $\mathrm{ms}$

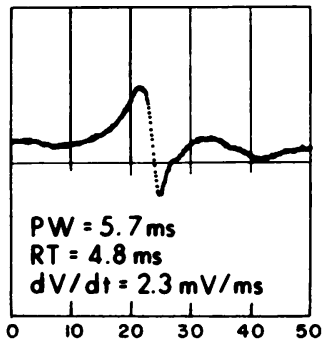

\subsection{Min after \\ Occlusion at Termination of Dysrhythmia}

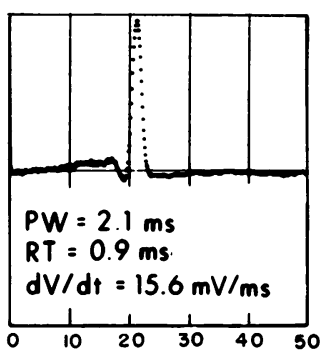

(n) 
tion) obtained from an Anapulse stimulator (W-P Instruments, Inc., New Haven, Conn.) was triggered by the $R$ wave of the surface ECG (Gould amplifier, Gould Inc.). The output of the Anapulse stimulator was connected to a photo-optically coupled, battery powered, current generator which was designed to supply a constant current (programmable from 1 to $99 \mathrm{~mA}$ ) at a voltage up to $150 \mathrm{~V}$, in $1 \mathrm{~mA}$ increments used to prevent ground-loop currents from affecting the current input at the electrode. The current generator was designed so that the current and voltage output delivered to the electrode could be monitored simultaneously through a battery-powered Tektronix oscilloscope (Tektronix, Inc., Beaverton, Oreg.). To induce premature beats, a 1-2-mA pulse was used for capture, and to induce ventricular fibrillation, a single pulse $(5 \mathrm{~ms})$ was delivered during the upstroke of the $T$ wave of the surface electrocardiogram. Initial stimuli were $30 \mathrm{~mA}$ with increases in 5-mA steps until ventricular fibrillation ensued. The amount of current required to induce ventricular fibrillation with a single pulse is greater than that required when trains of pulses are utilized (21). However, trains of pulses may cause release of norepinephrine from the heart (22), and might therefore influence cyclic nucleotide levels appreciably more.

To ascertain whether the changes in cyclic AMP could be attenuated by pharmacological $\beta$-receptor blockade, $d, l$ propranolol $(0.75 \mathrm{mg} / \mathrm{kg})$ was administered intravenously, over $5 \mathrm{~min}, 15 \mathrm{~min}$ before coronary occlusion. This dose is the minimum required to entirely antagonize the tachycardia induced by right stellate ganglion stimulation (bipolar platinum electrodes, Grass stimulator Grass Instruments Co., Quincy, Mass.). Similar dosages of $d, l$-propranolol have also been shown to be required to insure $\beta$-receptor blockade of circulating catecholamines (23).

Because anoxia increases both myocardial cyclic AMP (16-18) and adenosine release, two types of experiments were performed to determine whether adenosine, a potent stimulant of cyclic AMP accumulation in brain tissue (24), would stimulate cyclic AMP accumulation in the mammalian heart. In the first set of experiments, adenosine $(0.1 \mathrm{mM})$ was infused into the left anterior descending coronary artery via a 29-gauge needle. $90 \mathrm{~s}$ later, samples were taken from myocardium perfused with adenosine and from the lateral myocardium perfused by the circumflex coronary arterial blood (adenosine-free) and assayed for cyclic AMP. The high concentration of adenosine was chosen because adenosine is rapidly converted to inosine largely by adenosine deaminase in crythrocytes (25). Despite this phenomenon, the dose was sufficient to slow the ventricular rate in this preparation. In other experiments, isolated rabbit hearts perfused retrograde with Krebs-Henseleit bicarbonate at $37^{\circ} \mathrm{C}$ equilibrated with $95 \% \mathrm{O}_{2}, 5 \% \mathrm{CO}_{2}$ at $20 \mathrm{ml} / \mathrm{min}$ were studied so that adenosine could be administered at more physiological levels $(10 \mathrm{nM})$ in a perfusion medium without erythrocytes, before assay of cyclic AMP in fast-frozen samples.

\section{RESULTS}

The feline preparation utilized in the present experiments $(26,27)$ exhibits: (a) ventricular dysrhythmia with a consistent onset time $(2.4 \pm 0.3 \mathrm{~min})$, duration $(33.5 \pm 1.5 \mathrm{~min})$, and associated overall mortality $(20 \%)$ due to ventricular fibrillation; $(b)$ consistent frequency of premature ventricular complexes within the 1 st $h$ after occlusion $(1,205 \pm 97)$; $(c)$ a coronary arterial distribution very similar to that of man $(27) ;(d)$ consistent alterations in heart rate, systemic arterial pressure, cardiac output, and total peripheral resistance; and $(e)$
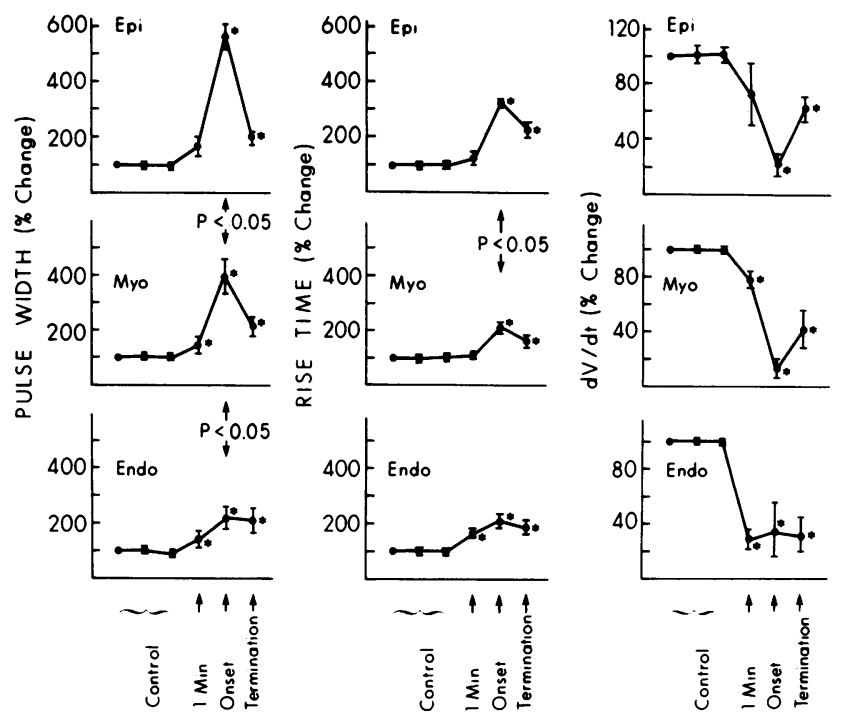

Figure 2 The combined data from 11 animals subjected to coronary occlusion. The figure illustrates changes in pulse width, rise time, and $\mathrm{dV} / \mathrm{d} t$ at three times during the control period before occlusion, the interval $1 \mathrm{~min}$ after occlusion, and immediately before the onset of the dysrhythmia, and the interval immediately before the termination of the dysrhythmia in epicardial (Epi), myocardial (Myo), and endocardial (Endo) layers of the ischemic zone. Values shown are percentage changes from average values in the control period $(100 \%)$ and are expressed as means \pm SD.

enhanced autonomic cardiac neural activity in the period immediately after coronary occlusion.

Electrophysiological alterations. Striking alterations were observed in electrograms from the ischemic area within minutes after occlusion (Fig. 1). In the absence of ischemia, electrograms remained stable with no significant alterations in width, rise time, velocity of upstroke or amplitude for $7 \mathrm{~h}$. Coronary occlusion resulted in a progressive decline in the velocity of upstroke with a corresponding increase in the rise time and pulse width in electrograms from ischemic sites only. The maximal changes in electrogram parameters in recordings from the ischemic zone coincided with the onset of ventricular dysrhythmia. Before termination of the dysrhythmia, partial but significant regression of the abnormalities was evident in all three parameters (Fig. 1).

The combined data from 11 such experiments in which animals recovered from the ventricular dysrhythmia are shown in Fig. 2. All values were standardized on the basis of $100 \%$ representing the initial control value. Coronary occlusion resulted in consistent and progressive increases in pulse width and rise time and a decrease in velocity of upstroke in the ischemic zones, with peak changes in each parameter occurring immediately before the onset of the ventricular dysrhythmia (Fig. 2). Myocardial and epicardial regions consistently demonstrated partial regression of abnormalities in all three electrogram parameters before the termination of the dysrhythmia. In contrast, 
endocardial regions of the ischemic zone exhibited continued depression of these parameters at termination of the dysrhythmia. Normal zone recordings failed to demonstrate any significant alterations in parameters after ischemia and during normal sinus rhythm.

A comparison of the changes in pulse width, rise time, and $\mathrm{dV} / \mathrm{d} t$ in the three levels of the ischemic zone at the onset of the dysrhythmia showed that the alterations in pulse width were greatest in epicardial areas and least prominent in endocardial regions (Fig. 2). Likewise, rise time was increased to a greater extent in epicardial compared to changes in myo- or endocardial areas. However, the velocity of upstroke was depressed comparably in all three regions of the ventricular wall at the time of onset of the dysrhythmia.

Regional changes in cyclic AMP content. In initial studies, simultaneous biopsies for assay of cyclic AMP were obtained from normal and central ischemic zones of the left ventricle from six cats at each selected time interval after coronary occlusion. Each experiment was "terminated immediately after" the biopsy was obtained. Coronary occlusion resulted in a significant increase in cyclic AMP in the ischemic zone $5 \mathrm{~min}$ later and cyclic AMP levels subsequently rose to peak value at $15 \mathrm{~min}$, returning to preocclusion levels by 30 min (Fig. 3). However, increases in cyclic AMP content in the normal zone were striking only during the 15-20 min after occlusion. Cyclic AMP in the central ischemic zones increased significantly compared to values in the normal zone $5,15,20,25,30$, and 35 min after occlusion (Fig. 3), suggesting a regional difference in localized adrenergic activity or possibly markedly decreased phosphodiesterase activity. The distribution of premature ventricular complexes (PVCs), ${ }^{1}(n=30$ different animals) at 5-min intervals after occlusion exhibits a progressive increase in PVC frequency which parallels closely the rise in cyclic AMP in the ischemic zone based on observations made in biopsies from other cats. It should be noted that the time of occurrence of peak level of cyclic AMP after coronary occlusion in the ischemic and normal zone precedes the time of occurrence of the peak PVC frequency (Fig. 3) suggesting not only that the two phenomena were temporally associated, but also that increased regional adrenergic activity reflected by increased cyclic AMP might have contributed to sustaining the dysrhythmia. However, this does not imply that increased regional adrenergic activity is necessary for initiation of the dysrhythmia. Indeed, when biopsies were obtained from seven other animals at the time of onset of the first abnormal beat, levels of cyclic AMP were not increased $(6.1 \pm 0.35$ and $6.4 \pm 0.30$ $\mathrm{pmol} / \mathrm{mg}$ protein in ischemic and normal zones, respec-

${ }^{1}$ Abbreviations used in this paper: PVCs, premature ventricular complexes; VF, ventricular fibrillation.

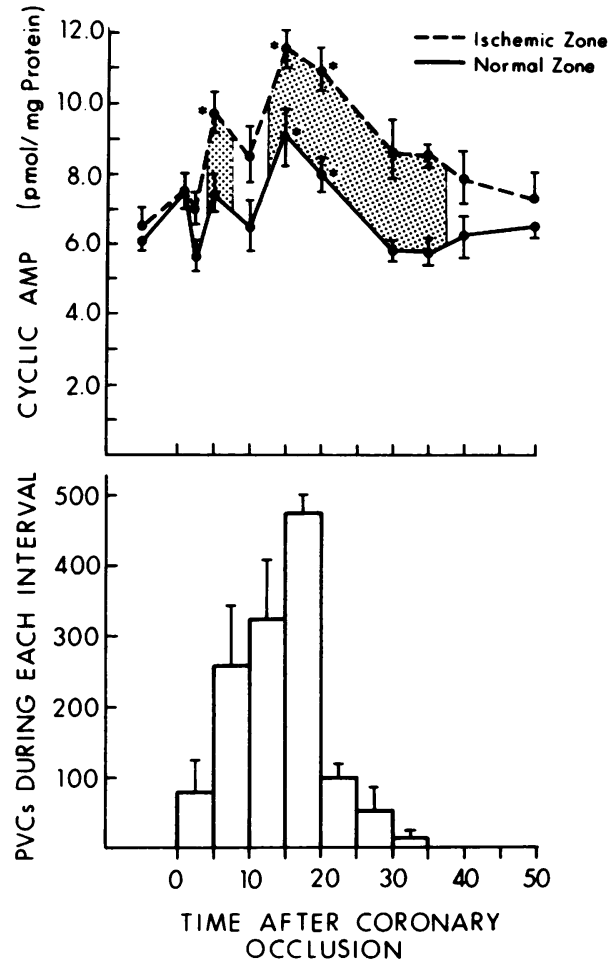

FIGURE 3 Changes in cyclic AMP (upper panel, $n=6$ cats at each point) in the ischemic and normal zones of the left ventricle at selected time intervals before and after coronary occlusion. The shaded areas indicate a significant difference $(P<0.05)$ between values from the ischemic and normal zones. The $\left(^{*}\right)$ indicates a significant difference from the control value. The frequency distribution of PVCs occurring during the same time intervals is illustrated in the lower panel ( $n=30$ cats). The vertical bars represent SE.

tively) suggesting that enhanced regional adrenergic activity and (or) accumulation of cyclic AMP were not necessary conditions for initiation of the dysrhythmia.

Effects of induced PVCs and induced ventricular fibrillation on regional cyclic AMP. Premature beats at selected coupling intervals were induced electrically in six nonischemic hearts for $15 \mathrm{~min}$ and the myocardium was subsequently sampled and assayed for cyclic AMP (Table I). Cyclic AMP levels did not increase even with a frequency of induced PVCs (552 \pm 53 ) in a 15 -min interval comparable to the spontaneous frequency after occlusion $(665 \pm 63)$. Likewise, electrical induction of PVCs for $15 \mathrm{~min}$ at a rate of $742 \pm 98$ in six animals, beginning $50 \mathrm{~min}$ after coronary occlusion when the spontaneous increase in cyclic AMP had abated, did not increase the cyclic AMP in normal or ischemic myocardium (Table I).

The data illustrated in Fig. 3 were obtained from 66 animals. An additional 13 animals succumbed to ventricular fibrillation within $2.5 \mathrm{~min}$ after coronary 
TABLE I

Influence of Induction of PVCs on Cyclic AMP in Ischemic and Nonischemic Hearts

Cyclic AMP

\begin{tabular}{c}
$\frac{\text { Cyclic AMP }}{$\cline { 2 - 3 } \text {$Anterior zone }}$ \\
\hline pmol/mg protein
\end{tabular}

Before occlusion

$6.4 \pm 0.5$

(6)

$5.7 \pm 0.3$

(6)

nonischemic hearts

After induced PVCs in hearts ischemic for $50 \mathrm{~min}$

$5.1 \pm 0.4$

(6)
$6.0 \pm 0.2$

$5.7 \pm 0.2$

$5.4 \pm 0.4$

Values are means $\pm \mathrm{SE}$.

Numbers in parentheses indicate numbers of animals in each group.

In the ischemic hearts, the zone of ischemia was anterior in each case.

occlusion and biopsies were obtained immediately thereafter (5-10 s). Results indicate that in those animals developing ventricular fibrillation (VF), cyclic AMP in both the central ischemic and normal zones was significantly $(P<0.01)$ elevated compared to changes in the corresponding zones at the same time after coronary occlusion (i.e. 2.0-min values in Fig. 3) in animals exhibiting PVCs but not VF (Fig. 4). In those animals developing VF, cyclic AMP in the ischemic zone was significantly elevated compared to values in normal zones in the same animals (Fig. 4). Although these results suggest that $\mathrm{VF}$ induced by myocardial

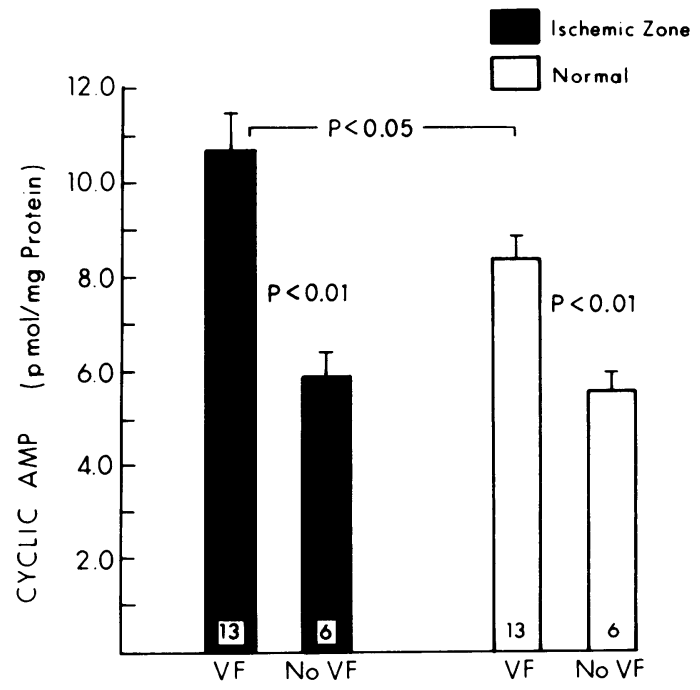

FIgURE 4 Cyclic AMP levels in ischemic and normal zones of the left ventricle in animals with and without spontaneous VF. The vertical bars represent the SEM and the numbers within the histograms indicate the numbers of animals in each group. ischemia is associated with increased regional adrenergic activity, VF per se might itself cause cyclic AMP to increase. However, when fibrillation was induced with a single pulse to the right ventricle, biopsies obtained within 5-10 s from normal $(n=5)$ and hearts subjected to coronary occlusion $(n=5)$ failed to exhibit increased cyclic AMP levels in either ischemic or normal zones (Table II). This lack of increase within the brief interval indicates that accumulation secondary to sympathetic reflex discharge requires a long interval after the onset of fibrillation. In addition, despite the marked differences in cyclic AMP in spontaneously fibrillating hearts after coronary occlusion, hemodynamic changes in animals with and without spontaneous fibrillation were comparable at the time corresponding to the interval before the rhythm disturbances (Table III, top two rows).

Effect of propranolol on cyclic AMP in ischemic and normal zones. To determine whether changes in efferent sympathetic activity accounted for the increases in cyclic AMP observed, we administered propranolol $(0.75 \mathrm{mg} / \mathrm{kg}$ i.v.) $15 \mathrm{~min}$ before ischemia in eight additional animals, a dose found previously to entirely block the increase in heart rate induced by isoproterenol injection $(0.5 \mu \mathrm{g} / \mathrm{kg})$ or right stellate ganglion stimulation in the cat $(9,23)$. Because the maximal increase in regional cyclic AMP occurred 15 min after coronary occlusion (Fig. 3), biopsies were obtained at this interval after occlusion in propranolol-treated animals. In the five of eight treated animals who survived for 15 min, significantly fewer PVCs occurred in the first 15 min after occlusion compared to control animals. The reduction in PVC frequency may be due in

TABLE II

The Influence of Induction of VF on Cyclic AMP in Ischemic and Nonischemic Hearts

\begin{tabular}{lcc}
\hline & \multicolumn{2}{c}{ Cyclic AMP } \\
\cline { 2 - 3 } & Anterior zone & Lateral zone \\
\hline & \multicolumn{2}{c}{ pmol/mg protein } \\
At the time of spontaneous VF & $10.7 \pm 0.8$ & $8.4 \pm 0.6$ \\
& $(13)$ & $(13)$ \\
After induced VF in & $4.8 \pm 0.6^{*}$ & $4.9 \pm 0.4^{*}$ \\
nonischemic hearts & $(5)$ & $(5)$ \\
After induced VF in hearts & $5.5 \pm 0.7^{*}$ & $6.1 \pm 0.2^{*}$ \\
ischemic for 50 min & $(5)$ & $(5)$ \\
\hline
\end{tabular}

Values are means $\pm \mathrm{SE}$

Numbers in parentheses indicate the number of animals in each group.

In the ischemic hearts, the zone of ischemia was anterior in each case.

${ }^{*} P<0.01$ compared to results in animals with spontaneous VF. 
TABLE III

Hemodynamic Alterations

\begin{tabular}{|c|c|c|c|c|c|}
\hline & \multirow{2}{*}{$\begin{array}{c}\text { Number } \\
\text { of cats }\end{array}$} & \multicolumn{2}{|c|}{ Before coronary occlusion } & \multicolumn{2}{|c|}{ 1.5 Min after coronary occlusion } \\
\hline & & Heart rate & Arterial pressure & Heart rate & Arterial pressure \\
\hline & & beats/min & $m m \mathrm{Hg}$ & beats/min & $m m \mathrm{Hg}$ \\
\hline Control* & 66 & $198 \pm 7.9$ & $105 \pm 8.2$ & $173 \pm 5.5 \ddagger$ & $88 \pm 9.1 t$ \\
\hline VF $\S$ & 13 & $204 \pm 9.2$ & $111 \pm 4.1$ & $181 \pm 6.1 t$ & $92 \pm 10.2 \$$ \\
\hline Propranolol" & 5 & $150 \pm 4.1$ & $96 \pm 10.2$ & $128 \pm 7.5 \ddagger$ & $78 \pm 10.1 \ddagger$ \\
\hline Propranolol and VF $\$$ & 3 & $141 \pm 1.2$ & $103 \pm 7.1$ & $122 \pm 7.6 \ddagger$ & $84 \pm 11.2 \ddagger$ \\
\hline
\end{tabular}

Numbers are mean \pm SE.

Propranolol refers to animals treated with this drug as described in the text.

* Animals with coronary occlusion who were not treated with propranolol.

f $\boldsymbol{P}<\mathbf{0 . 0 5}$ with paired comparisons between the pre- and postocclusion period.

$\S$ Animals with hearts biopsied at the moment of spontaneously occurring VF.

"Animals with hearts biopsied 15 min after coronary occlusion.

part to the corresponding decrease in sinus rate with propranolol treatment. In addition, myocardial cyclic AMP content at this time was significantly reduced (Fig. 5). The control values were obtained $15 \mathrm{~min}$ after occlusion (Fig. 3) and compared to values in biopsies obtained at corresponding intervals after occlusion in animals treated with propranolol. Nevertheless, three animals treated with propranolol did develop spontaneous VF within 3 min after coronary occlusion. All

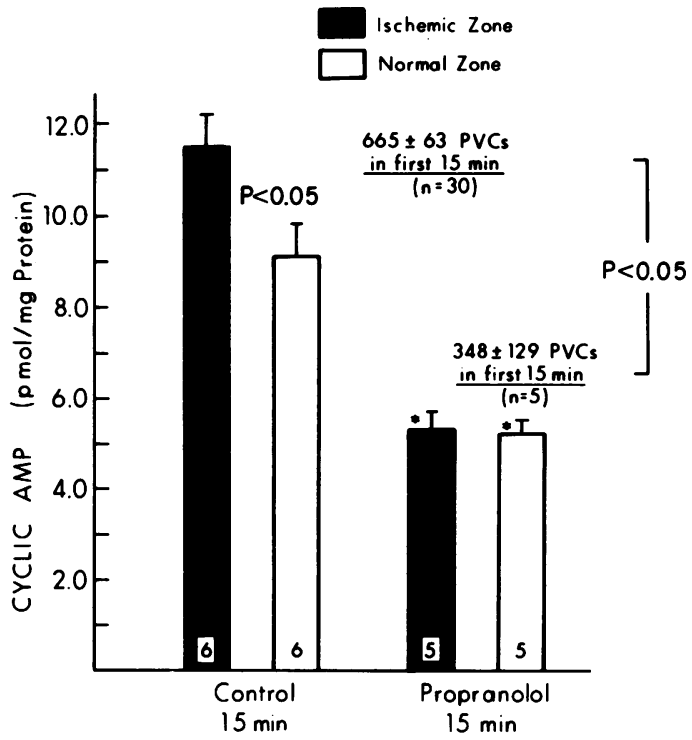

FIGURE 5 Cyclic AMP levels in ischemic and normal zones of the left ventricle $15 \mathrm{~min}$ after coronary occlusion in control animals (left) and animals treated with propranolol (right) who survived for at least $15 \mathrm{~min}$. The numbers of PVCs in the control (top) and treated animals (bottom) are indicated. The $\left.{ }^{*}\right)$ indicates a significant decrease in the cyclic AMP levels in propranolol-treated compared to control animals $(P<0.01)$. Vertical bars represent SEM and the numbers within the histograms indicate the number of animals in each group. three exhibited a significant increase in cyclic AMP within ischemic zones, compared to values before occlusion in animals treated with propranolol (Fig. 6). Although the absolute levels of cyclic AMP were reduced by propranolol, the increase from control values in animals who developed VF was not precluded by $\beta$-receptor blockade (Fig. 7). Furthermore, although propranolol significantly reduced the sinus rate before coronary occlusion, the alterations in systemic arterial pressure and heart rate induced by ischemia were similar in treated animals with and without VF (Table III).

At least three possibilities might account for the association of ventricular fibrillation with increased cyclic AMP in animals treated with propranolol: $(a)$ adequate pharmacological $\beta$-blockade, as assessed by

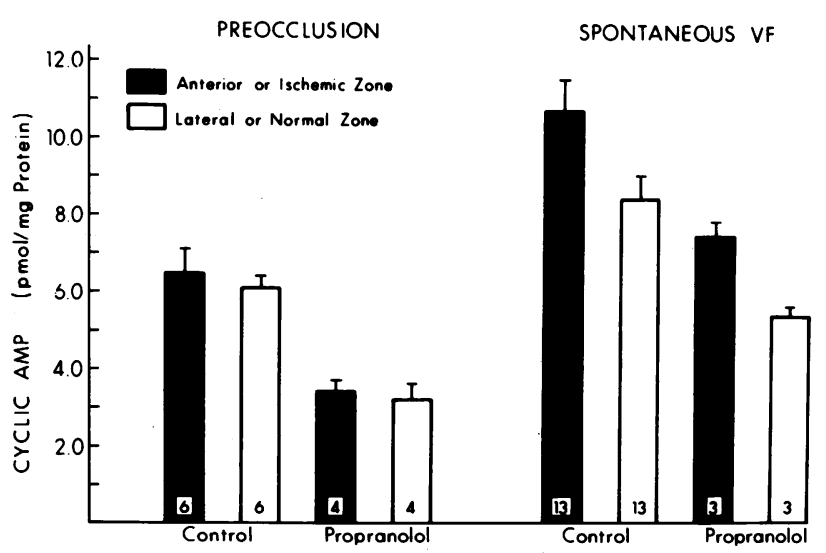

FIGURE 6 Cyclic AMP levels in anterior (ischemic zones) or lateral (normal zones) of the left ventricular wall in the interval before occlusion (left side) and at the time of onset of spontaneous VF (VF-right side) in control animals and cats treated with propranolol. Vertical bars represent SEM and the numbers within the histograms indicate the number of animals in each group. 


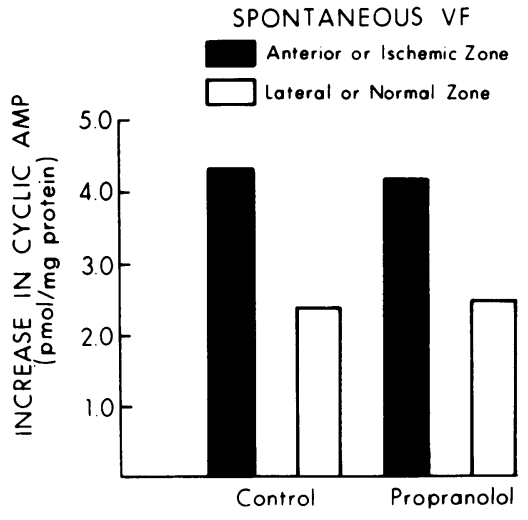

FIGURE 7 Changes in cyclic AMP from values before occlusion, at the onset of $\mathrm{VF}$ in anterior (ischemic) and lateral (normal) zones of the left ventricle in control cats and animals treated with propranolol. The numbers within the histograms indicate the number of animals in each group.

inhibition of changes in sinus rate, does not attenuate all efferent sympathetic nerve traffic to the heart (10, $11) ;(b)$ effects of the intramyocardial release of norepinephrine subsequent to ischemia (28) may not be blocked by pharmacological blockade; and $(c)$ mechanisms other than $\beta$-receptor stimulation by catecholamines may be responsible for increased cyclic AMP seen in animals treated with propranolol who developed VF. One possibility in this category, adenosine release, was readily excluded. Adenosine, known to be a potent stimulant of adenylate cyclase in the central nervous system (24) and to be released with myocardial ischemia (29), did not alter the cyclic AMP levels in ischemic or nonischemic cat hearts in vivo $(0.1 \mathrm{mM})$ or isolated rabbit hearts $(10 \mathrm{nM})$ (Table IV). Thus, it is unlikely that release of adenosine accounted for the increase in cyclic AMP during ischemia.

\section{DISCUSSION}

Electrophysiological alterations. The bipolar electrogram recordings in the present study were used to assess local electrophysiological changes occurring at the time of onset and offset of ventricular dysrhythmia. The bipolar electrogram reflects temporal dispersion throughout the population of cells in the region of the electrode, as well as the configuration of action potentials from individual cells (30). In ischemic zones of the left ventricular wall, profound alterations in width, rise time, and velocity of upstroke of the bipolar electrogram in endo-, epi-, and mid-myocardial layers occurred before the onset of dysrhythmia possibly due to several factors including: $(a)$ decreased rate of rise and amplitude of individual transmembrane action potentials with or without altered conduction velocity; (b) asynchronous depolarization of cells within the region surrounding the electrode; and $(c)$ a change in the direction of spread of impulse with respect to the poles of the recording electrode altering the overall amplitude of the electrogram recorded. The dramatic increase in pulse width in recordings from ischemic zones before the onset of dysrhythmia supports the concept that cells in the region of the electrode were depolarizing asynchronously because of decreased membrane responsiveness and (or) delayed conduction due to heterogeneous depression of cells within the ischemic zone. The consistent time-dependent alterations in electrogram parameters soon after the onset of ischemia argues against the possibility that directional changes in wave fronts with respect to the poles of the recording electrode were entirely responsible, because these would be random. It appears that the most likely basis for the early changes in bipolar electrograms induced by ischemia is a decrease in conduction velocity within the ischemic zone due to decreased resting membrane potential and consequent suppression of amplitude and rate of rise of transmembrane action potentials of individual cells. Although studies have utilized bipolar electrogram recordings, primarily from epicardial regions, to characterize ventricular dysrhythmias induced by ischemia $(1,2,5)$, quantitative changes have not been characterized by automated analysis of large numbers of sequential electrograms.

In the present study, recordings from endocardial regions of the ischemic zone did not exhibit depressed electrogram parameters comparable to those in epicardial or myocardial recordings before the onset of dysrhythmia, suggesting that the electrophysiological alterations in endocardial areas were less profound.

TABLE IV

The Influence of Adenosine on Cyclic AMP in Cat Hearts in situ and Isolated Rabbit Hearts

\begin{tabular}{|c|c|c|c|}
\hline & & $\begin{array}{l}\text { Anterior } \\
\text { zone }\end{array}$ & $\begin{array}{l}\text { Lateral } \\
\text { zone }\end{array}$ \\
\hline \multirow{3}{*}{ Cat } & & \multicolumn{2}{|c|}{$\begin{array}{c}\text { Cyclic AMP } \\
\text { pmol/mg protein }\end{array}$} \\
\hline & Control & $\begin{array}{c}6.4 \pm 0.5 \\
(6)\end{array}$ & $\begin{array}{c}6.0 \pm 0.2 \\
(6)\end{array}$ \\
\hline & $\begin{array}{l}\text { Adenosine } \\
(0.1 \mathrm{mM})\end{array}$ & $\begin{array}{c}7.2 \pm 0.8 \\
(3)\end{array}$ & $\begin{array}{c}5.9 \pm 0.3 \\
(3)\end{array}$ \\
\hline \multirow{3}{*}{ Rabbit } & & \multicolumn{2}{|c|}{$\begin{array}{c}\text { Whole heart cyclic AMP } \\
\text { pmol/mg protein }\end{array}$} \\
\hline & Control & \multicolumn{2}{|c|}{$\begin{array}{c}4.20 \pm 0.4 \\
(4)\end{array}$} \\
\hline & $\begin{array}{r}\text { Adenosine } \\
(10 \mathrm{nM})\end{array}$ & \multicolumn{2}{|c|}{$\begin{array}{c}4.30 \pm 0.3 \\
(4)\end{array}$} \\
\hline
\end{tabular}

Values are means $\pm \mathrm{SE}$.

Numbers in parentheses indicate the number of hearts in each group. 
Oxygenation, substrate supply, or washout of noxious metabolites in intraventricular cavitary blood may reduce the coronary perfusion requirements of Purkinje fibers in endocardial regions and may contribute to their protection (31).

Delay in activation within the ischemic zone has been correlated with the occurrence of ventricular dysrhythmia $(1,2,5)$. The present study was designed to characterize the nature of depolarization of cells in the ischemic zone by analyzing quantitatively the initial deflections in electrograms recorded at the time of occurrence and cessation of dysrhythmia rather than focusing on the temporal pattern of activation. At the time of onset of dysrhythmia, the abnormalities detected in regional electrograms reached a maximum, and this, coupled with their regression before cessation of dysrhythmia suggests that the regional electrophysiological changes detected contributed to the dysrhythmia.

Regional adrenergic activity. In the present study, regional alterations in cyclic AMP content were utilized as a potential index of local adrenergic input. Catecholamine stimulation of the heart is accompanied by increases in cyclic AMP (12-14). Furthermore, administration of dibutyryl cyclic AMP $(32,33)$ and ionotophoretic injection of cyclic AMP intracellularly (34) produce electrical alterations in isolated cardiac tissue similar to those evoked by catecholamines. Nevertheless, factors such as local accumulation due to decreased washout or phosphodiesterase inhibition may directly or indirectly increase cyclic AMP levels. Decreased washout seems unlikely to account for the increase because the halflife of cyclic AMP in tissues is so short and because persistent flow (10-20\% of normal) is typical in zones of infarction. In the present study, we observed complete attenuation by $\beta$-receptor blockade of the large increase in cyclic AMP 15 min after coronary occlusion. Furthermore, the dose of propranolol utilized was sufficient for $\beta$-adrenergic blockade assessed by attenuation of the tachycardia induced by isoproterenol or right stellate ganglion but insufficient to exert quinidine-like membrane effects (35). Thus, it appears likely that the changes in cyclic AMP did reflect catecholamine activation of cardiac $\beta$-receptors. Other possibilities such as inhibition of phosphodiesterase appear to be less likely because catecholamines inhibit phosphodiesterase only at very high levels (15) and because propranolol blocks the increase of cyclic AMP so effectively.

Results of this study implicate an increase in regional adrenergic activity in the perpetuation but not the initiation of dysrhythmia after coronary occlusion based on: (a) the progressive increase of cyclic AMP in the ischemic zone preceding the progressive increase in frequency of PVCs; $(b)$ the failure of in- duced PVCs to alter regional cyclic AMP; and (c) effective attenuation by propranolol of the rise in cyclic AMP 15 min after occlusion with simultaneous depression of PVCs. This interpretation is compatible with findings in pharmacological studies with propranolol (9) with results in dogs exhibiting diminished dysrhythmia induced by ischemia after ganglionectomy (36).

In the present study, cyclic AMP was consistently elevated in the ischemic compared to the corresponding normal zone. Prior treatment with propranolol abolished not only the rise in cyclic AMP $15 \mathrm{~min}$ after occlusion but also the disparity between cyclic AMP levels in ischemic and normal zones. This disparity may be important because heterogeneous, adrenergic neural stimulation of the heart is more arrhythmogenic than uniform, humoral stimulation (37). The heterogeneity of cyclic AMP levels in the present study may reflect many factors including: $(a)$ local intramyocardial, non-neurally mediated release of norepinephrine confined to ischemic regions; or $(b)$ persistence of catecholamines in ischemic zones because of impaired metabolism, reuptake, or washout.

The first possibility is supported by findings in isolated perfused hearts devoid of efferent sympathetic input, demonstrating that anoxia results in rapid increases in cyclic AMP $(13,16-18)$ that can be inhibited by $\beta$-receptor blockade $(13,17)$. Increases in extracellular potassium secondary to ischemia (38) may release intramyocardial catecholamines (39). The released norepinephrine may originate from peripheral sympathetic nerve endings since administration of tyramine $30 \mathrm{~min}$ before anoxia precludes the increase in catecholamines seen with anoxia (40). The second possibility, regarding washout, would not account for the decreased cyclic AMP seen relatively late after ischemia, but no direct evidence is yet available to support or refute it.

Among the animals in whom ventricular fibrillation developed after coronary occlusion, cyclic AMP in the ischemic and normal zones was significantly elevated compared to corresponding values in hearts with only premature ventricular beats. Because electrical induction of ventricular fibrillation failed to alter the cyclic AMP levels in either normal or ischemic hearts, it appears likely that the increased regional efferent sympathetic activity elicited the increased cyclic AMP and contributed to, rather than resulted from, VF. No demonstrable differences were noted in hemodynamics after coronary occlusion in animals who developed VF compared to those who did not.

$\beta$-Receptor blockade with propranolol did not attenuate the incidence of VF, the magnitude of the increase in cyclic AMP in normal or ischemic zones of the left ventricle (despite the lower initial values), or the disparity in cyclic AMP levels between the two zones in 
those hearts that did fibrillate. In animals with fibrillation despite treatment with propranolol, the increase in cyclic AMP was large, even though the baseline value was depressed and the peak absolute value was also depressed (Fig. 6). These observations suggest that factors other than adrenergic stimulation could be responsible for the increases in cyclic AMP in these particular hearts, or that $\beta$-receptor blockade does not attenuate all efferent sympathetic nerve traffic to the heart (10). Ephaptic transmission, independent of chemical neurotransmitters, may be one contributing factor. Furthermore, because VF in animals not treated was preceded by large increases in cyclic AMP (Fig. 4), it seems probable that in propranolol-treated animals developing VF, complete blockade would not occur due to the competitive nature of the antagonist.

Studies have demonstrated that clamping or transection of the aorta in dogs $(41,42)$ or in rats $(18,43)$ increases cyclic AMP, an effect abolished by $\beta$ receptor blockade $(18,41-43)$; and anoxia increases rat heart cyclic AMP (16-18) also blocked by $\beta$-receptor blockade $(17,18)$. However, these changes have not been related previously to electrophysiological events subsequent to ischemia. A recent editorial (44) has implicated cyclic AMP itself in the genesis of VF. The present study presents direct evidence supporting the hypothesis that cyclic AMP or the processes responsible for its accumulation such as regionally enhanced adrenergic activity in ischemic zones contribute to the maintenance of ventricular dysrhythmia after myocardial ischemia and to the occurrence of $\mathrm{VF}$.

\section{ACKNOWLEDGMENTS}

The authors wish to thank Mr. Gerard Clarke and Ms. Elaine Carlson for their expert technical assistance and Ms. Carolyn Lohman for preparation of the manuscript.

This work was supported in part by National Institutes of Health grant HL 17646, Specialized Center of Research in Ischemic Heart Disease; and Missouri Heart Association grant-in-aid.

\section{REFERENCES}

1. Scherlag, B. J., R. H. Helfant, J. I. Haft, and A. N. Damato. 1970. Electrophysiology underlying ventricular arrhythmias due to coronary ligation. Am. J. Physiol. 219: 1665-1671.

2. Scherlag, B. J., N. El-Sherif, R. Hope, and R. Lazzara. 1974. Characterization and localization of ventricular arrhythmias resulting from myocardial ischemia and infarction Circ. Res. 35: 372-383.

3. Lazzara, R., N. El-Sherif, and B. J. Scherlag. 1973. Electro-physiological properties of canine Purkinje cells in one-day-old myocardial infarction. Circ. Res. 33: $722-734$.

4. Lazzara, R., N. El-Sherif, and B. J. Scherlag. 1974. Early and late effects of coronary artery occlusion on canine Purkinje fibers. Circ. Res. 35: 391-399.
5. Corr, P. B., and B. E. Sobel. 1977. Automated data processing. An essential decision-making aid in the treatment of acute myocardial infarction. Adv. Cardiol. 20: $54-71$.

6. Webb, S. W., A. A. J. Adgey, and J. F. Pantridge. 1972. Autonomic disturbance at onset of acute myocardial infarction. Br. Med. J. 3: 89-92.

7. Ebert, P. A., R. B. Vanderbeek, R. J. Allgood, and D. C. Sabiston, Jr. 1970. Effect of chronic cardiac denervation on arrhythmias after coronary artery ligation. Cardiovasc. Res. 4: 141-147.

8. Khan, M. I., J. T. Hamilton, and G. W. Manning. 1972. Protective effect of beta adrenoceptor blockade in experimental coronary occlusion in conscious dogs. Am. J. Cardiol. 30: 832-837.

9. Corr, P. B., and R. A. Gillis. 1975. Effect of autonomic neural influences on the cardiovascular changes induced by coronary occlusion. Am. Heart J. 89: 766-774.

10. Gillis, R. A., D. L. Pearle, and T. Hoekman. 1974. Failure of beta-adrenergic receptor blockade to prevent arrhythmias induced by sympathetic nerve stimulation. Science (Wash. D. C.). 185: 70-72.

11. Govier, W. C. 1968 . Myocardial alpha adrenergic receptors and their role in the production of positive inotropic effect by sympathomimetic agents. J. Pharmacol. Exp. Ther. 159: 82-90.

12. Sobel, B. E., and S. E. Mayer. 1973. Cyclic adenosine monophosphate and cardiac contractility. Circ. Res. 32: 407-413.

13. Wollenberger, A. 1975. The role of cyclic AMP in the adrenergic control of the heart. In Contraction and Relaxation in the Myocardium. W. G. Nayler, editor. Academic Press, Inc., New York. 113-190.

14. Drummond, G. I., and S. J. Hemmings. 1973. Role of adenylate cyclase-cyclic AMP in cardiac actions of adrenergic amines. Recent Adv. Stud. Card. Struct. Metab. 3: 213-222.

15. Goren, E. N., and O. M. Rosen. 1972. Inhibition of a cyclic nucleotide phosphodiesterase from beef heart by catecholamines and related compounds. Mol. Pharmacol. 8: 380-384.

16. O'Brien, J. A., and R. C. Strange. 1975. The release of adenosine $3^{\prime}: 5^{\prime}$-cyclic monophosphate from the isolated perfused rat heart. Biochem. J. 152: 429-432.

17. Shahab, L., A. Wollenberger, E. G. Krause, and S. Genz. 1972. Effect of Acute Ischaemia on Myocardial Function. M. F. Oliver, D. G. Julian, and K. W. Donald, editors. Churchill-Livingstone, Edinburgh.

18. Dobson, J. G., and S. E. Mayer. 1973. Mechanisms of activation of cardiac glycogen phosphorylase in ischemia and anoxia. Circ. Res. 33: 412-420.

19. Lowry, O. H., N. J. Rosebrough, A. L. Farr, and R. J. Randall. 1951. Protein measurement with the folin phenol reagent. J. Biol. Chem. 193: 265-275.

20. Steiner, A. L., R. E. Wehmann, C. W. Parker, and D. M Kipnis. 1972. Radioimmunoassay for the measurement of cyclic nucleotides. Adv. Cyclic Nucleotide Res. 2: 51-61.

21. Tamargo, J., B. Moe, and G. K. Moe. 1975. Interaction of sequential stimuli applied during the relative refractory period in relation to determination of fibrillation threshold in the canine ventricle. Circ. Res. 37: 534-541.

22. Vincenzi, F. F., and T. C. West. 1963. Release of autonomic mediators in cardiac tissue by direct subthreshold electrical stimulation. J. Pharmacol. Exp. Ther. 141: 185-194. 
23. Nayler, W. G., and J. Tay. 1972. Effect of o-2-hydroxy3-(tertbutylamino) propoxybenzonitrile $\mathrm{HCl}$ (KO 1366) on beta adrenergic receptors in cardiovascular system. $J$. Pharmacol. Exp. Ther. 180: 302-316.

24. Schultz, J. 1975. Cyclic adenosine $3^{\prime}, 5^{\prime}$-monophosphate in guinea-pig cerebral cortical slices: studies on the role of adenosine. J. Neurochem. 24: 1237-1242.

25. Caen, J. P., C. S. P. Jenkins, H. Michel, J. J. Chivot, S. Levy-Toledano, and F. Rendu. 1973. Adenosine metabolism in platelets and plasma. Ser. Haematol. 6: 317-332.

26. Corr, P. B., and R. A. Gillis. 1974. Role of the vagus nerves in the cardiovascular changes induced by coronary occlusion. Circulation. 49: 86-97.

27. Corr, P. B., D. L. Pearle, J. R. Hinton, W. C. Roberts, and R. A. Gillis. 1976. Site of myocardial infarction. A determinant of the cardiovascular changes induced in the cat by coronary occlusion. Circ. Res. 39: 840-847.

28. Braasch, W., S. Gudbjarnason, P. S. Puri, K. G. Ravens, and R. J. Bing. 1968. Early changes in energy metabolism in the myocardium following acute coronary artery occlusion in anesthetized dogs. Circ. Res. 23: 429-438.

29. Berne, R. M. 1964. Regulation of coronary blood flow. Physiol. Rev. 44: 1-29.

30. Brooks, C. M., B. F. Hoffman, E. E. Suckling, and O. Orias. 1955. Excitability of the Heart. Grune and Stratton, Inc., New York. 124-130.

31. Wit, A. L., and P. L. Friedman. 1975. The basis for ventricular arrhythmias accompanying myocardial infarction: alterations in electrical activity of ventricular muscle and Purkinje fibers after coronary artery occlusion. Arch. Intern. Med. 135: 459-472.

32. Tsien, R. W., W. Giles, and P. Greengard. 1972. Cyclic AMP mediates the effects of adrenaline on cardiac Purkinje fibres. Nat. New Biol. 240: 181-183.

33. Kobayashi, T., R. Nakayama, and K. Kimura. 1971. Effects of glucagon, prostaglandin $\mathrm{E}_{1}$ and dibutyryl cyclic 3',5'-AMP upon the transmembrane action potential of guinea pig ventricular fiber and myocardial contractile force. Jpn. Circ. J. 35: 807-819.
34. Tsien, R. W. 1973. Adrenaline-like effects of intracellular ionotophoresis of cyclic AMP in cardiac Purkinje fibres. Nat. New Biol. 245: 120-122.

35. Barrett, A. M. 1969. A comparison of the effect of ( \pm )propranolol and $(+)$-propranolol in anesthetized dogs; $\beta$ receptor blocking and haemodynamic action. J. Pharm. Pharmacol. 21: 241-247.

36. Fowlis, R. A. F., C. T. M. Sang, P. M. Lundy, S. P. Ahuja, and H. Colhoun. 1974. Experimental coronary artery ligation in conscious dogs six months after bilateral cardiac sympathectomy. Am. Heart J. 88: 748-757.

37. Han, J., P. Garcia de Jalon, and G. K. Moe. 1964. Adrenergic effects on ventricular vulnerability. Circ. Res. 14: $516-524$

38. Harris, A. S. 1966. Potassium and experimental coronary occlusion. Am. Heart J. 71: 797-802.

39. Borda, L., R. Shuchleib, and P. D. Henry. 1977. Effects of potassium on isolated coronary arteries: Modulation of adrenergic responsiveness and release of norepinephrine. Circ. Res. 41: 778-786.

40. Wollenberger, A., and L. Shahab. 1965. Anoxia-induced release of noradrenaline from the isolated perfused heart. Nature (Lond.). 207: 88-89.

41. Wollenberger, A., E. G. Krause, and G. Heier. 1969. Stimulation of $3^{\prime}, 5^{\prime}$-cyclic AMP formation in dog myocardium following arrest of blood flow. Biochem. Biophys. Res. Commun. 36: 664-670.

42. Rabinowitz, B., W. W. Parmley, M. Kligerman, J. Norman, S. Fujimura, S. Chiba, and J. M. Matloff. 1975. Myocardial and plasma levels of adenosine $3^{\prime}: 5^{\prime}$-cyclic phosphate. Studies in experimental myocardial ischemia. Chest. 68: 69-74.

43. Limas, C. J., D. Ragan, and E. D. Fries. 1974. Effect of acute cardiac overload on intramyocardial cyclic $3^{\prime}, 5^{\prime}$ AMP: relation to prostaglandin synthesis. Proc. Soc. Exp. Biol. Med. 147: 103-105.

44. Podzuweit, T., W. F. Lubbe, and L. H. Opie. 1976. Cyclic adenosine monophosphate, ventricular fibrillation and antiarrhythmic drugs. Lancet. I: 341-342. 